\title{
AN AXISYMMETRIC FINITE VOLUME FORMULATION FOR CREEP ANALYSIS
}

\author{
K. Zarrabi \\ School of Mechanical and Manufacturing Engineering \\ The University of New South Wales \\ Sydney 2052 \\ AUSTRALIA \\ A. Basu \\ School of Mechanical and Manufacturing Engineering \\ The University of New South Wales \\ Sydney 2052 \\ AUSTRALIA
}

\begin{abstract}
A new finite volume algorithm for computing elastic-creep stress distributions within pressure components that have axisymmetric geometries and subjected to axisymmetric loading is presented. The formulation of a system of simultaneous equations from equilibrium, constitutive and boundary equations of the finite volume elements that are used to model the component is described in details. An iterative technique is used to solve the resulted simultaneous equations and compute the displacement fields at various time points from which the strain and stress fields are computed. The method is verified against analytical solutions and applied to internally corroded/eroded tubes. It is also shown that the proposed finite volume method is more accurate than the finite element method that is formulated on the similar bases.
\end{abstract}

Key Words: Finite Volume, Creep, Axisymmetric Pressure Components

\section{NOMENCLATURE}

\begin{tabular}{|c|c|}
\hline$a_{i} \cdot h_{i}$ & Linear coefficients used in equations (12) \\
\hline$f_{1} \cdot f_{2}$ & Creep functions used in equations (14) \\
\hline h & A factor defined in equation ( 16$)$ \\
\hline$l$ & Half of the boiler tube length \\
\hline$"$ & Creep stress index \\
\hline$r_{t}$ & Radial coordinate \\
\hline $\mathrm{t}$ & Time \\
\hline u. $w$ & Radial and axial displacement components respectively \\
\hline$z_{1}$ & Axial coordinate \\
\hline B & Creep stress coefficient \\
\hline$B_{1},\left(i_{i}, l\right)_{1}, H_{l},\left(i_{i}, H H_{1}, l_{t}\right.$ & Coefficients used in equations (14) \\
\hline $\mid \prime) \mid$ & Elasticity matrix \\
\hline
\end{tabular}




$E$
$F$
$P$
$P_{e}$
$P_{e x t}$
$S_{\theta}=\frac{\sigma_{\theta}}{p_{e x t}}$
$\alpha_{1}, \alpha_{2}$
$\beta$
$\left\{\varepsilon^{c}\right\}$
$\left\{\varepsilon^{e}\right\}$
$\left\{\varepsilon^{t}\right\}$
$\left\{\varepsilon^{i}\right\}=\left\{\varepsilon_{r}^{i}, \varepsilon_{\theta}^{i}, \varepsilon_{z}^{\prime}, \gamma_{r=}^{i}\right\}^{T}$
$\left\{\dot{\varepsilon}^{c}\right\}$
$\dot{\varepsilon}_{e f f}^{c}$
$\left\{\sigma^{c}=\left\{\sigma_{r}, \sigma_{\theta}, \sigma_{z}, \tau_{r z}\right\}^{T}\right.$
$\sigma_{e f f}$
$v$
$\tau=E p_{e x t}^{(n-1)}$,
$\theta$

Young's modulus

von Mises Potential function

Uniform internal pressure

Axial traction

Radial traction

Non-dimensional hoop stress

Factors defined in equations (15) and (17) respectively

Angle that an element side on the bounday makes with the radial axis

Creep strain components

Elastic strain components

Total strain components

Strain components

Creep strain rate components

von Mises creep effective strain rate

Stress components

von Mises effective stress

Poisson ratio

Non-dimensional time

Hoop coordinate

\section{INTRODUCTION}

The finite element method (FEM) has dominated the field of numerical pressure vessel stress analysis for the past 30 years or more. However, an alternative approach based upon the finite volume method (FVM), originally developed by the computational fluid dynamics community, is now being applied to certain specific problems in design and analysis of pressurised components such as internally thinned boiler tubes. Unlike FEM, in which the relevant conservation principle, equilibrium of forces, is only satisfied in a global sense, FVM is conservative in that it guarantees that the elements used to model the component are themselves in equilibrium and tractions are continuous across inter element boundaries.

Recently, a number of FVMs have been developed. Ivankovic and Williams (1995) have applied FVM to study the elastic behaviour of cracked components subjected to dynamic loading. Onate et al (1993) have suggested a formulation for two-dimensional elastic problems. Zarrabi and Basu (1999) have developed a new FVM for axisymmetric components when materials restricted to elastic deformation. There are a number of other finite volume formulations as well. 
This paper presents a new finite volume formulation for elastic-creep analysis of pressure components with axisymmetric geometries and loading. To the authors knowledge no such formulation has been developed before. The verification of proposed formulation, its comparison with a simialar finite element formulation and its application to boiler tubes are also presented.

\section{AXISYMMETRIC FINITE VOLUME FORMULATION FOR ELASTIC-CREEP STRESS ANALYSIS}

It is assumed that the material temperature is constant and uniform. Although the following formulation is general and can be applied to any axisymmetric problem that is subjected to elastic-creep deformation, it is specifically developed to apply to internally corroded/eroded boiler tubes (Figure 1).

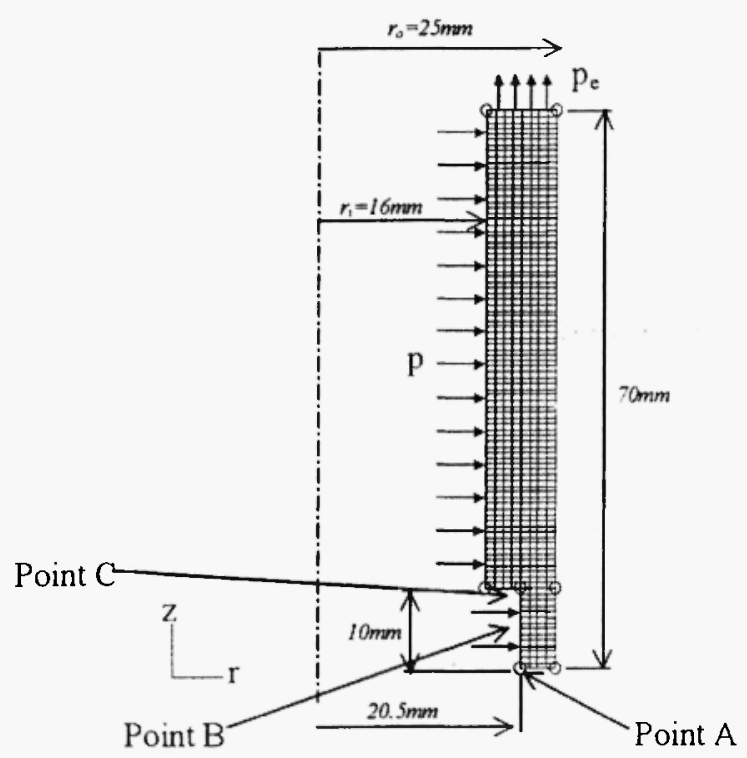

Figure 1 - Finite volume model of internally eroded/corroded tube subject to a uniform internal pressure (p) and end pressure $\left(p_{e}\right)$

\section{Constitutive Relationships}

For an elastic-creep material, the total strain components, $\left\{\varepsilon^{t}\right\}$, is the sum of the elastic, $\left\{\varepsilon^{e}\right\}$, and creep $\left\{\varepsilon^{c}\right\}$ strain components:

$\left\{\varepsilon^{t}\right\}=\left\{\varepsilon^{e}\right\}+\left\{\varepsilon^{c}\right\}$ 
where $\left\{\varepsilon^{t}\right\}=\left\{\varepsilon_{r}^{t}, \varepsilon_{\theta}^{t}, \varepsilon_{z}^{t}, \gamma_{r=}^{i}\right\}^{T}$ with $i=t, e, c$ and $r, \theta$ and $z$ refer to the radial, hoop and axial directions respectively. Using Hooke's law:

$\{\sigma\}=[D]\left\{\varepsilon^{e}\right\}$

where $\left\{\sigma_{\}}\right\}=\left\{\sigma_{r}, \sigma_{\theta}, \sigma_{z}, \tau_{r}\right\}$ is the stress vector, and $[D]$ is the elasticity matrix, i.e.

$[D]=\frac{E}{(1+v)(1-2 v)}\left[\begin{array}{cccc}(1-v) & v & v & 0 \\ v & (1-v) & v & 0 \\ v & v & (1-v) & 0 \\ 0 & 0 & 0 & \frac{(1-2 v)}{2}\end{array}\right]$

with $E$ and $v$ are Young's modulus and Poisson's ratio respectively. Combining Eqs (1) to (3) gives:

$\{\sigma\}=\mid l) \mid\left(\left\{\varepsilon^{i}\right\}-\left\{\varepsilon^{c}\right\}\right)$

Creep strain components can be determined from:

$\left\{z^{c}\right\}=\int\left\{z^{c}\right\} d t$

where, is time and the creep strain rate components are derived from:

$\left\{\dot{\varepsilon}^{c}\right\}=\stackrel{c}{\dot{\varepsilon}_{e f f}} \frac{\partial F}{\partial\{\sigma\}}$

with $F$ is the von Mises potential function and $\dot{\varepsilon}_{e f f}^{c}=B \sigma_{, f f}^{n}$ is the effective von Mises creep strain rate. Note that $B$ and $n$ are material parameters and $\sigma_{o f f}$ is the von Mises effective stress. Note that, we have assumed material deforms according to secondary creep only. The extension of the present formulation to include primary and tertiary creep will be reported in the future publications.

\section{Equilibrium Equations and Discretisation}

Figure 2 shows an axisymmetric finite volume element (i.e., FV1) together with some of its surrounding elements (i.e., FV2, .., FV4). The differential equilibrium equations are:

$$
\left\{\begin{array}{l}
\frac{\partial \sigma_{r}}{\hat{C} r}+\frac{\partial \tau_{r z}}{\hat{C} z}+\frac{u_{r}-\sigma_{\theta}}{r}=0 \\
\frac{\hat{\partial} \tau_{r z}}{\hat{C} r}+\frac{\partial \sigma_{z}}{\hat{c} z}+\frac{\tau_{r z}}{r}=0
\end{array}\right.
$$




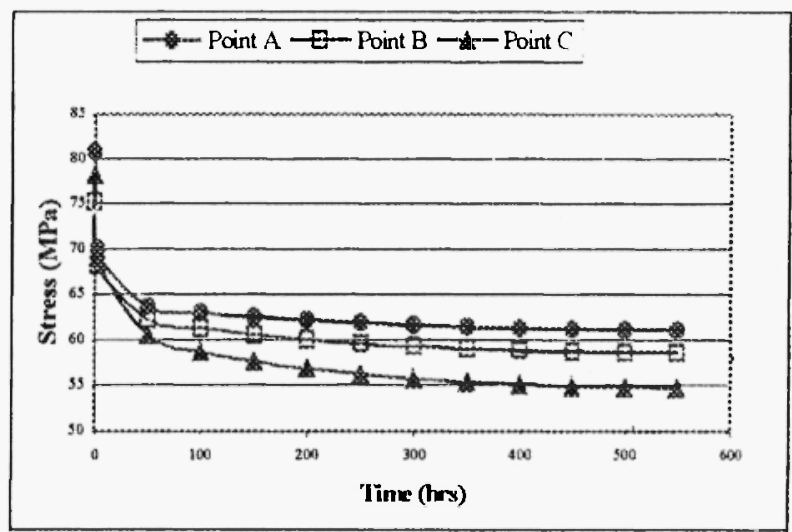

Figure 2 - An axisymmetric finite volume element (FV 1) and its surrounding elements: FV2, FV3, FV 4 , FV5.

Integrating equations (7) over the volume of element FVl will give:

$$
\left\{\begin{array}{l}
\iint_{\mathrm{JJ}} 2 \pi r \frac{\partial \sigma_{r}}{\partial r} d r d z+\iint_{\mathrm{J}} 2 \pi r \frac{\partial \tau_{r z}}{\partial z} d r d z+\iint 2 \pi r \frac{u_{r}-\sigma_{\theta}}{r} d r d z=0 \\
\iint 2 \pi r \frac{C \tau_{r z}}{\partial r} d r d z+\iint 2 \pi r \frac{\partial \tau_{z} z}{\partial z} d r d z+\iint 2 \pi r \frac{r_{r z}}{r} d r d z=0
\end{array}\right.
$$

Using the divergence theorem, equations (8) can be rewritten in the following form:

$$
\left\{\begin{array}{l}
r \oint \sigma_{r} d z+\oint r \tau_{r} d r-\iint \sigma_{0} d r d z=0 \\
r \oint \tau_{r} d z+\oint r \sigma_{z} d r=0
\end{array}\right.
$$

Now considering equations (9), the first and second terms in the first equation and the terms in the second equations represent integrals around the boundary of FVI and the last term in the first equation represents the sum of hoop stresses over the face of FV1. To integrate equations (9), one needs to know the variations of various stress components with $r$ and $z$. The present formulation assumes that the stress components along each element side remain constant and are allocated to mid-position on each side. This allows equations (9) to be integrated to obtain: 


$$
\left\{\begin{array}{l}
\left(z_{2}-z_{1}\right)\left(\frac{r_{1}+r_{2}}{2}\right) \sigma_{r 1}+\left(r_{1}-r_{2}\right)\left(\frac{r_{1}+r_{2}}{2}\right) \tau_{r z 1}+\left(z_{3}-z_{2}\right)\left(\frac{r_{2}+r_{3}}{2}\right) \sigma_{r 2}+\left(r_{2}-r_{3}\right)\left(\frac{r_{2}+r_{3}}{2}\right) \tau_{r=2} \\
\quad+\left(z_{4}-z_{3}\right)\left(\frac{r_{3}+r_{4}}{2}\right) \sigma_{r 3}+\left(r_{3}-r_{4}\right)\left(\frac{r_{3}+r_{4}}{2}\right) \tau_{r z 3}+\left(z_{1}-z_{4}\right)\left(\frac{r_{4}+r_{1}}{2}\right) \sigma_{r 4}+\left(r_{4}-r_{1}\right)\left(\frac{r_{4}+r_{1}}{2}\right) \tau_{r z 4} \\
-\frac{A}{4}\left(\sigma_{\theta 1}+\sigma_{\theta 2}+\sigma_{\theta 3}+\sigma_{\theta 4}\right)=0 \\
\left(r_{1}-r_{2}\right)\left(\frac{r_{1}+r_{2}}{2}\right) \sigma_{z 1}+\left(z_{2}-z_{1}\right)\left(\frac{r^{\prime}+r_{2}}{2}\right) \tau_{r z 1}+\left(r_{2}-r_{3}\right)\left(\frac{r_{2}+r_{3}}{2}\right) \sigma_{z 2}+\left(z_{3}-z_{2}\right)\left(\frac{r_{2}+r_{3}}{2}\right) \tau_{r z 2} \\
\quad+\left(r_{3}-r_{4}\right)\left(\frac{r_{3}+r_{4}}{2}\right) \sigma_{z 3}+\left(z_{4}-z_{3}\right)\left(\frac{r_{3}+r_{4}}{2}\right) \tau_{r z 3}+\left(r_{4}-r_{1}\right)\left(\frac{r_{4}+r_{1}}{2}\right) \sigma_{z-4}+\left(z_{1}-z_{4}\right)\left(\frac{r_{4}+r_{1}}{2}\right) \tau_{r z 4}=0
\end{array}\right.
$$

where $r_{i}$ and $z_{l}$ with $i=1.2,3,4$ are the radial and axial coordinates of each corner node of element FVl, $\sigma_{\theta i}, \sigma_{n}, \sigma_{z l}$, and $\tau_{r z 1}$ are the stress components at the mid-position of each side of element FVl, and $A$ is the face area of element FVl. By combining equations (4) with equations ( 10 ), the equilibrium equations will be expressed in terms of strain components. But the strain components at the mid-side position are related to the radial and axial displacements $(u, w)$ and their gradients; for example, assuming small strain conditions, for side 1 :

$$
\left\{\begin{array}{l}
\varepsilon_{\mathrm{r} 1}^{\mathrm{t}}=\left(\frac{d u}{d r}\right)_{1} \\
\varepsilon_{\theta 1}^{t}=\left(\frac{u}{r}\right)_{1} \\
\left.r_{z=1}^{t}=: \frac{d w}{d z}\right)_{1} \\
\gamma_{r=1}^{t}=\left(\frac{d u}{d z}\right)_{1}+\left(\frac{d w}{d r}\right)_{1}
\end{array}\right.
$$

Similar equations to equations (11) can be written for sides 2, 3, and 4 of element FVl. Now, the present formulation assumes that $"$ and $w$ vary bilinearly between the centroids of adjacent element faces so that.

$$
\left\{\begin{array}{l}
u=a_{1}+a_{2} r+a_{3} z+a_{4} r z \\
w=h_{1}+b_{2} r+b_{3} z+b_{4} r z
\end{array}\right.
$$

where $a_{i}$ and $h_{t}$ with $i=1.2 .3 .4$ are constants that can be determined in terms of the coordinates of the centroids of four adjacent elements. Equations (12) are then differentiated to obtain the displacement gradients. Therefore, by combining equations (4) and (10) to (12), the equilibrium equations can be written in terms of the unknown displacements at the centroids of the element faces. Note that for each centroid there are two equilibrium equations with two unknown displacement components, $u_{t}$ and $w_{t}$. 


\section{Boundary Conditions}

On the boundary, the unknowns are the radial and axial displacement components ( $u_{b l}$, $\left.w_{b_{1}}\right)$ at the mid-position of each element side. The following boundary conditions are considered. For free surfaces: $u_{b i} \neq 0, w_{b i} \neq 0$. For symmetry about the $r$-axis:

$u_{b l} \div \therefore, w_{b l}=0, \tau_{b r i}=0$ where $\tau_{b r i i}$ is the shear stress component with respect to the $r z$ axes acting at the mid-side boundary node $\iota$. For sides subjected to pressure $p_{l}$ :

$$
\left\{\begin{array}{l}
\sigma_{n t}=\sigma_{b z i} \operatorname{Cos}^{2} \beta+\sigma_{b r i} \operatorname{Sin}^{2} \beta-\tau_{b r z} \operatorname{Sin}(2 \beta)=p_{t} \\
\tau_{t}=-\frac{1}{2}\left(\sigma_{b r t}-\sigma_{b z t}\right) \operatorname{Sin}(2 \beta)-\tau_{b r z l}\left(\operatorname{Cos}^{2} \beta-\operatorname{Sin}^{2} \beta\right)=0
\end{array}\right.
$$

where $\beta$ is the angle that the loaded side makes with the $r$-axis, $\sigma_{n t}, \tau_{i}$ are the normal and shear stresses at the mid-side of each loaded side respectively, and $\sigma_{b z i}, \sigma_{b r i}, \tau_{b r z i}$ are the axial, radial, and shear stress components at the mid-side of each loaded side respectively. Equations (13) are obtained using the well-known stress transformations or Mohr's circle. It is assumed that the stress comonents are constant along the element sides that are positioned on the boundary. This assumption is consistant with previously stated assumptions for elements sides that are not located on the boundary. The above boundary conditions produce two equations for each mid-side node that is positioned on the boundary together with two unknown displacement components.

\section{Assembly}

By combining equilibrium, constitutive and boundary equations as explained above, the following set of simultaneous equations will be obtained:

$$
\left\{\begin{array}{l}
\sum_{i=1}^{N_{1}} B_{i} u_{i}+\sum_{i=1}^{N_{1}} C_{i} w_{i}=\sum_{i=1}^{N_{i}} f_{1}\left(G_{i}\left\{\varepsilon^{c^{c}}\right\}\right) \ldots, \ldots \\
\sum_{i=1}^{N_{2}} D_{i} u_{b i}+\sum_{i=1}^{N_{2}} F_{i} w_{b i}=\sum_{i=1}^{N_{2}} P_{i}+\sum_{i=1}^{N_{2}} f_{2}\left(H_{i}\left\{\varepsilon^{c}\right\}\right)
\end{array}\right.
$$

where $N_{1}$ is the total number of elements, $N_{2}$ is the total number of element sides that are on the boundary, $f_{1}$ and $f_{2}$ are functions that include creep strain components. The coefficients $B_{t}, C_{1}, D_{t}, F_{t}, G_{t}, H_{t}$ and $P_{i}$ will depend on the element geometry, material properties at operating temperature and applied pressure. Equations (14) represent $2\left(N_{1}+N_{2}\right)$ simultaneous non-linear equations; the sources of non-linearity are functions $f_{1}$ and $f_{2}$. Note that equations (14) have less equations than those obtained from an assembly of a similar mesh consisting of 4-node axisymmetric finite elements. This is because while in the present formulation there are two equilibrium equations for the centroid of each element face, in a displacement-based finite element formulation there are eight equilibrium equations ( 2 for each corner node). The boundary equations for the 
present formulation are also less than those for a counterpart finite element mesh. Equations (14) have, however, the same degree of sparsity as those obtained from assembly of 4-node axisymmetric finite elements. Therefore, the CPU-time required to solve equations (14) and the computer storage requirements for the present finite volume formulation are expected to be less than those required for the similar finite element formulation

\section{Solution Algorithm}

There are a number of solution techniques available to solve a set of non-linear simultaneous equations system such as that defined by equations (14). For the sake of simplicity, the present study employs a fully explicit algorithm and it is as follows. Initially when $t=0$, there is no creep strain and therefore equations (14) becomes linear $\left(f_{1}\left(\left\{\varepsilon^{c}\right\}\right)=0, f_{2}\left(\left\{\varepsilon^{c}\right\}\right)=0\right)$ and they will be solved as an elastic problem to obtain displacements, stresses and total strains. Note that at time zero, elastic and total strains are equal. Then the computed stresses are used in equation (6) to compute the creep strain rates at time zero. Then creep strains at the end of a small time interval are computed using equation (5). It is assumed that the time interval is sufficiently small so that during this time interval the creep strain rates remain constant. Knowing the creep strains at the end of the time interval $\left(f_{1}\left(\left\{\varepsilon^{c}\right\}\right)=\right.$ Cons $\tan t, f_{2}\left(\left\{\varepsilon^{c}\right\}\right)=$ Cons $\left.\tan \iota\right)$, equations (14), that again become linear, are solved to compute new displacements and stresses and total strains at the end of the time interval. Then the new stresses are used again to compute creep strain rates for the next small time interval. The algorithm is continued until a specified time is reached. The success of this algorithm depends on the proper selection of the time interval $\left(\Delta_{i}\right)$ for each iteration $i$ so it merits brief description.

\section{Time Interval}

It is well known that a fully explicit time integration scheme is conditionally stable (Taylor et al, 1995 and Owen and Hinton, 1980). Therefore, in order to achieve a valid solution, limits must be placed on the time interval. During the initial, or transient, states of creep, the stresses change rapidly. It is necessary to choose exceptionally small time intervals in this region. As the solution approaches its steady-state, larger time intervals may be applied.

A combination of the several schemes has been employed to ensure stability and solution accuracy (Zarrabi and Hosseini-Toudeshky, 1995). Firstly, the magnitude of the time interval in each iteration $\left(s_{1}\right)$ is controlled by a user-defined factor $\alpha_{1}$ according to:

$$
N_{i} \leq \alpha_{1}\left[\frac{\varepsilon_{e f f, t}^{t}}{\left\{\dot{\varepsilon}^{c}\right\}_{i}}\right]_{\min }
$$

An initial estimate of the value of the time interval is made by evaluating the above expression for the mid-point of each element side throughout the mesh and selecting the 
minimum value. Numerical experimentation suggests that for explicit procedures $0.01<\alpha_{1}<0.1$.

A second scheme limits the change in time intervals between any two intervals according to

$$
\Delta_{t+1}<k \Delta \Delta_{t}
$$

where $k$ is a user-defined constant and usually has values in the range $1<k<1.5$.

The final scheme controls the time interval by a factor $\alpha_{2}$. This factor limits the maximum effective creep strain increment $\left(\Delta \varepsilon_{e f f, i}^{c}\right)$ as a fraction of the effective elastic strain $\left(\varepsilon_{e f f}^{e},\right)$, implying that:

$$
\Delta t_{i} \leq \alpha_{2}\left[\frac{\varepsilon_{\text {eff, } i}^{e}}{\dot{\varepsilon}_{\text {eff }, i}^{c}}\right]_{\min }
$$

Once again, equation (17) is evaluated for each element side throughout the mesh and the minimum is taken. Numerical experimentation suggests that: $0<\alpha_{2}<1.0$. The final value of the time interval must satisfy equations (15) to (17).

\section{VERIFICATION}

Three problems have been used to verify the model and algorithm described above. These are described below.

\section{Axially Loaded Bar}

The bar is depicted in Figure 3 and had a solid circular cross-sectional area with a radius of $r=100 \mathrm{~mm}$ and a length of $l=500 \mathrm{~mm}$. The uniform axial traction was $P_{e}=100 \mathrm{MPa}$.

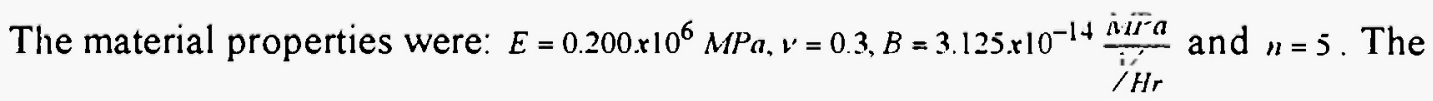
finite volume model of the bar had a $4 \times 4$ mesh. Figure 4 shows the variation of the effective total strain at a point in the bar with time computed using FVM. Figure 4 also depicts the analytical solution. It is apparent that any differences between the two solutions are negligible.

\section{Disk with a Circular Hole Subjected to Uniform Radial External Traction}

The disk containing a circular hole is shown in Figure 5 with: $a=16 \mathrm{~mm}$, $l:=0.200 . \times 10^{6} \Lambda I P^{3} a, \quad v=0.3 . \quad n=3 . \quad B=1 \frac{M M^{\prime} a}{1 / n r}$ and was loaded externally by a uniform traction, $p_{c u t}=1 \mathrm{M} / \mathrm{I}^{\prime}$, in the radial direction. Taking into account the axial symmetry of 


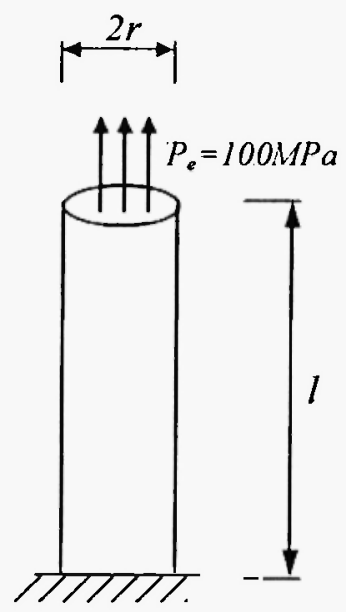

Figure 3 - Axially loaded bar.

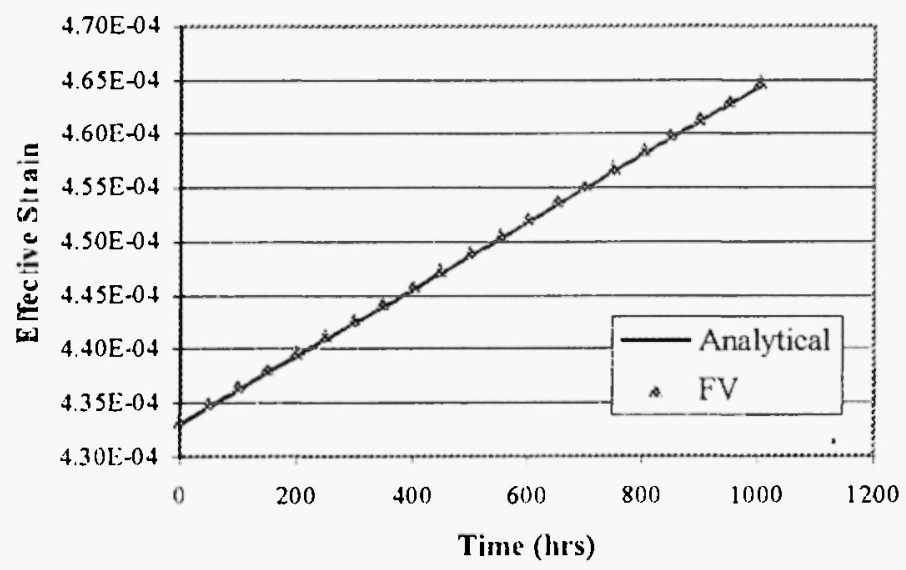

Figure 4 - Comparison of finite volume and analy tical solutions for effective total strain obtained for axially loaded bar.

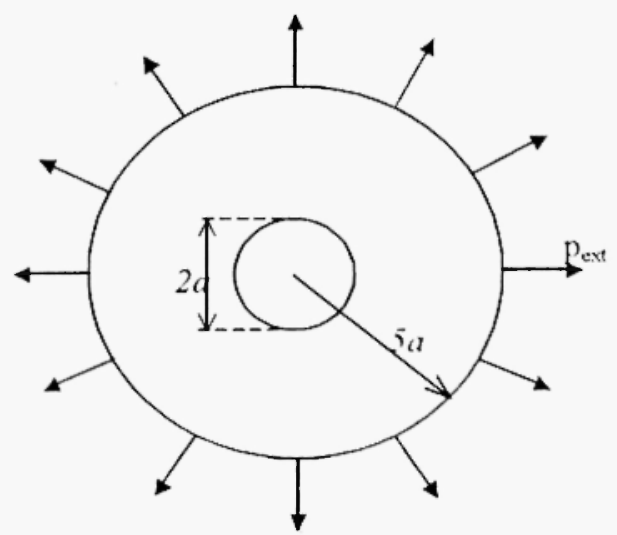

Figure 5 - Disk subjected to uniform radial external traction. 
the problem, a typical finite volume mesh of the disk is shown in Figure 6. The hoop stress at the edge of the hole (Point $A$ in Figure 6 ) computed using three different meshes are compared with the analytical solution in Figure 7 . The non-dimensional hoop stress and time depicted in Figure 7 are defined as $S_{\theta}=\frac{\pi_{\hat{\nu}}}{p_{\text {ext }}}$ and $\tau=E p_{\text {ext }}^{(n-1)} /$ respectively. The results depicted in Figure 7 show that as the mesh became finer, the computed results became closer to the analytical results so that for $32 \times 8$ mesh (i.e., Curve 3 in Figure 7 ) the maximum error was $8.5 \%$.

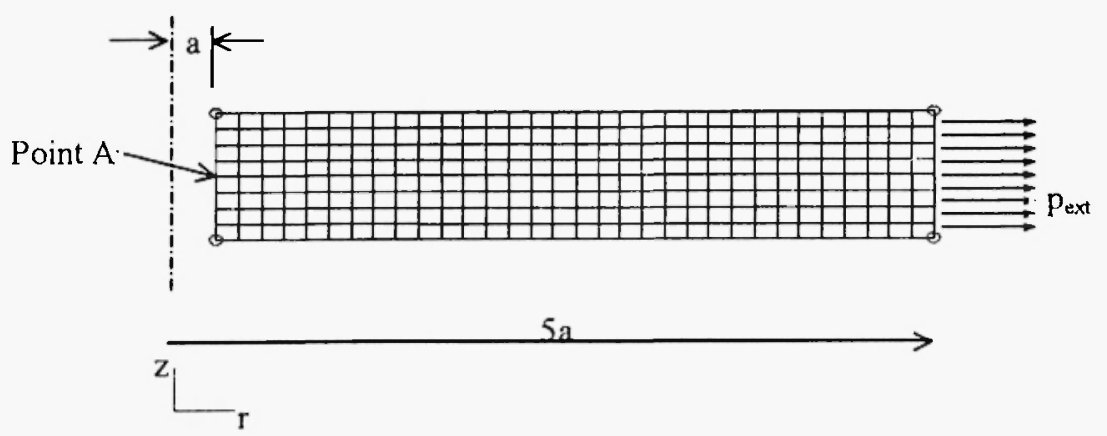

Figure 6 - Finite volume mesh of a disk subjected to a uniform radial external traction.

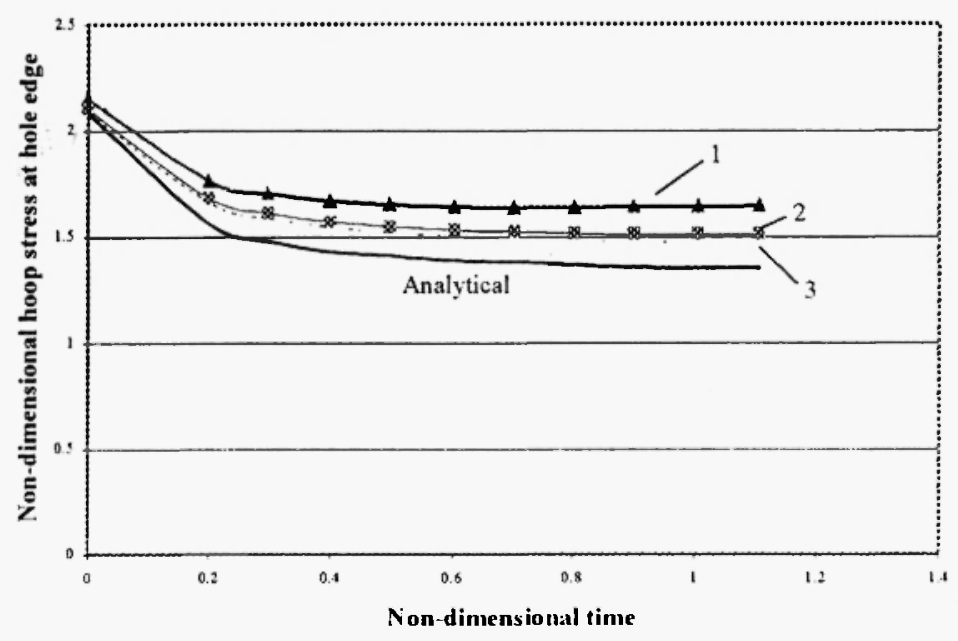

Figure 7 - Variation of non-dimensional hoop stress at the hole edge with time; Cune $1-8 \times 2$ mesh, Cune $2-16 x+$ mesh, Curve $3-32 \times 8$ mesh. 


\section{Steady-State Solution for a Uniform Thick-wall Tube}

The finite volume model of the thick-wall tube is shown in Figure 8. It was subjected to a uniform internal pressure of $p=20 \mathrm{MPa}$ and end pressure of $p_{e}=13.875 \mathrm{MPa}$. The material properties where: $E=0.200 \times 10^{6} M P a, v=0.3, B=3.125 \times 10^{-14} \frac{i \frac{i \bar{F} a}{i / H r} .}{i / H} . n=5$. The computed elastic and steady-state stresses in the hoop and radial directions are compared with the corresponding analytical solutions in Figure 9 . Figure 9 shows that the differences between the two stress values were negligible.

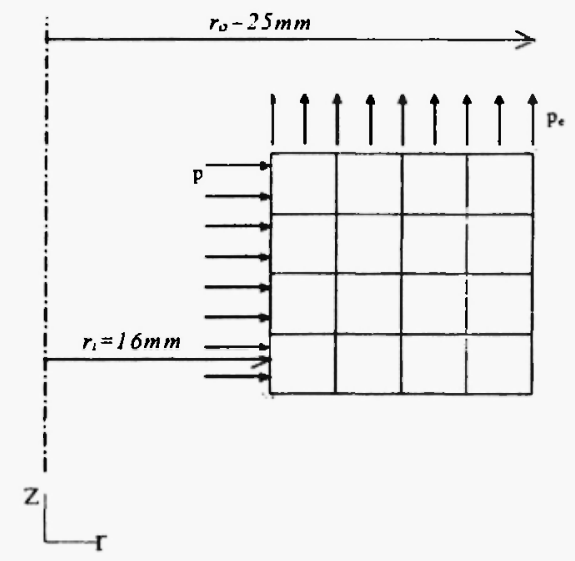

Figure 8 - Finite volume mesh of a thick-wall tube subjected to uniform internal pressure $p$ and end pressure $p_{e}$

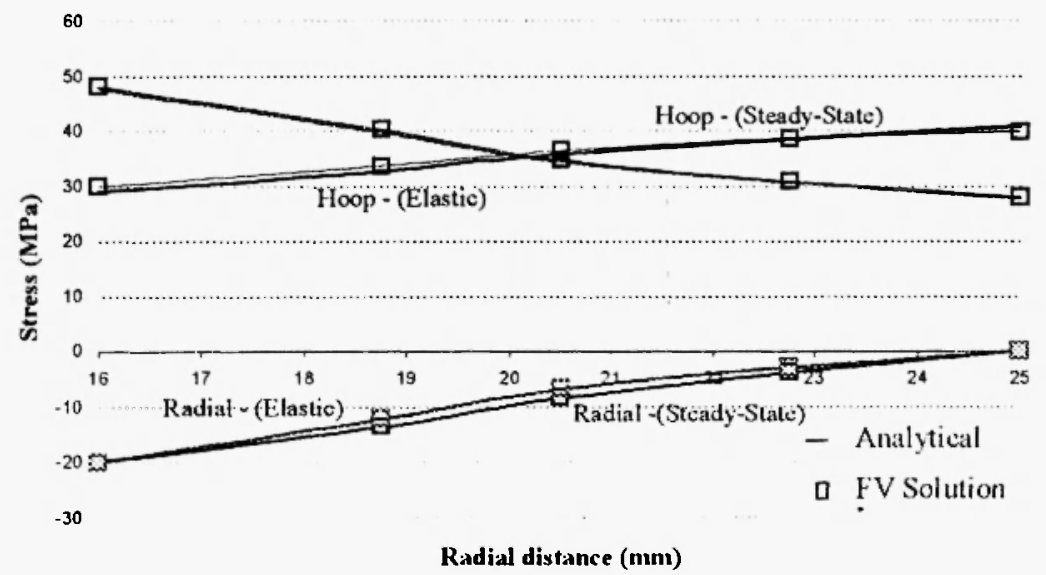

Figure 9 - Comparison of finite volume and analytical solutions for va rious stress components obtained for uniform thick-wall tube. 


\section{APPLICATION TO INTERNALLY ERODED/CORRODED BOILER TUBE}

The internally eroded/corroded tube shown in Figure 1 was modelled using 728 axisymmetric finite volume elements. It was assumed that the eroded/corroded area had a rectangular cross-sectional area. Because of symmetry about the centre-line of the eroded/corroded area, only half of the length of the tube was modelled. The loading and material properties were the same as those for the thick-wall tube described above. The variation of computed von Mises stresses at strategic Points A, B, and C (Figure 1) with time are shown in Figure 10. At time zero, due to stress concentration, the elastic stress at Point $C$ is higher than those at Points $A$ and $B$. Stresses redistribute and after a short period of time, stresses at Points $A$ and $B$ become less than that at Point $C$ with Point $C$ having the smallest stress.

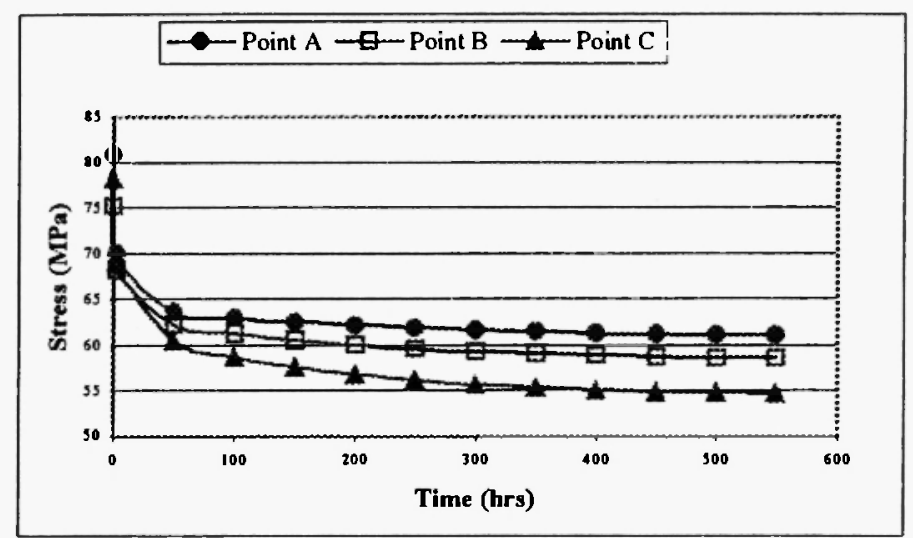

Figure 10 -Variation of Von-Mises Stress at Point A, Point B and Point C with time for internally eroded/corroded tube.

\section{COMPARISON OF FINITE VOLUME AND FINITE ELEMENT METHODS}

The three problems that were used to verify our FVM above were also employed below to compare our FVM with a similarly formulated FEM (Zarrabi and Hosseini-Toudeshky, 1995). That is, similar to our FVM, the FEM used 4-node quadilateral elements with time stepping and integration algoriuthms similar to those of our FVM.

\section{Axially Loaded Bar}

The material properties, dimensions and loading for the bar were as before (Figure 3 ). The coarsest possible mesh for the FVM was a $2 \times 2$ mesh. Therefore, it was decided to generate $2 \times 2$ meshes for both FVM and FEM. The computed axial stresses by FVM and FEM were equal to the analytical solution $\left(100 \omega / T^{\prime}\right)$ everywhere, i.e., no error was detected. 


\section{Disk with a Circular Hole Subjected to Uniform Radial External Traction}

The material properties, dimensions and loading for the disk containing a circular hole were as before (Figure 5). Taking into account the axial symmetry of the problem, $32 \times 8$ meshes were generated for both FVM and FEM (Figure 6). The non-dimensional hoop stress at the edge of the hole (Point A in Figure 6), $s_{\theta}=\frac{\sigma_{A}}{p_{\text {ext }}}$, computed using FVM and FEM are compared with the analytical solution in Figure 11. The results depicted in Figure 11 show that: (1) the computed stresses by FVM and FEM have the same distribution as that predicted by the analytical solution, (2) for the same level of mesh density, FVM maximum error (8.5\%) was less than that for FEM (39.6\%).

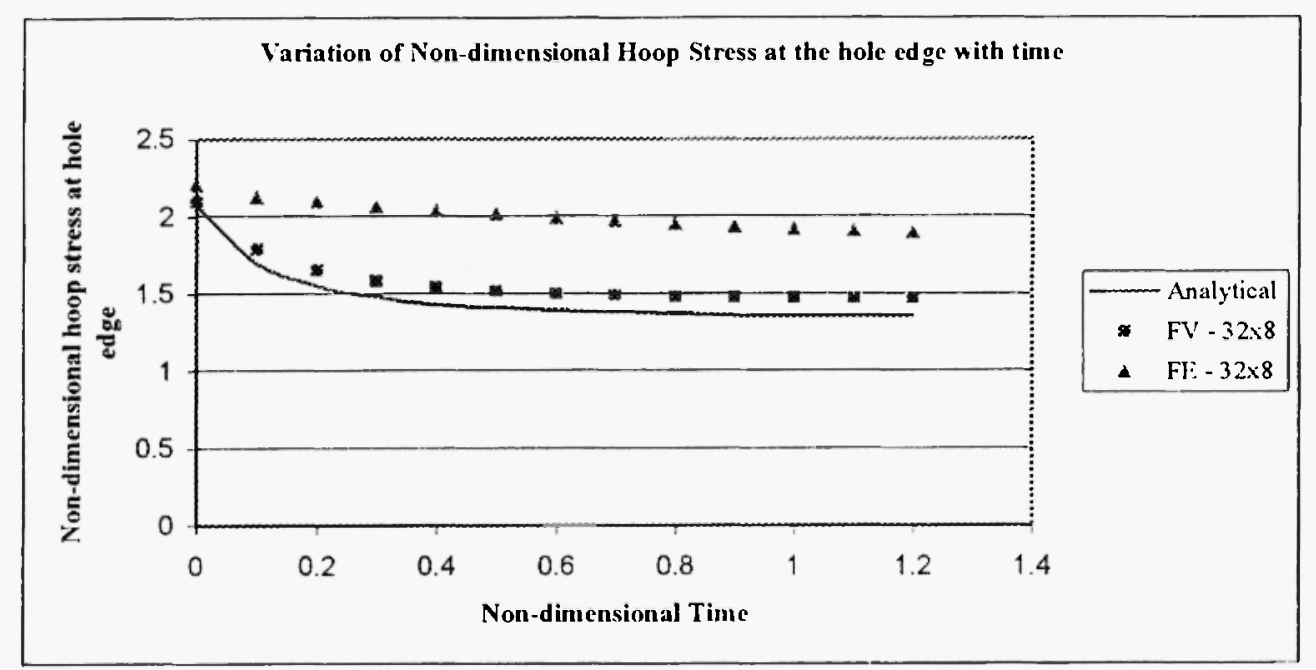

Figure 11 - Comparison of computed stresses using FVM and FEM for disk.

\section{Steady-State Solution for a Uniform Thick-wall Tube}

The material properties, dimensions and loading were the same as before. A $4 \times 4$ mesh that was used for both FVM and FEM is shown in Figure 8. The computed steady-state stresses in the hoop direction are compared with the corresponding analytical solution in Figure 12. The maximum error in computed hoop stress using FVM was $4.0 \%$ whereas that in using FEM was $5.4 \%$. 


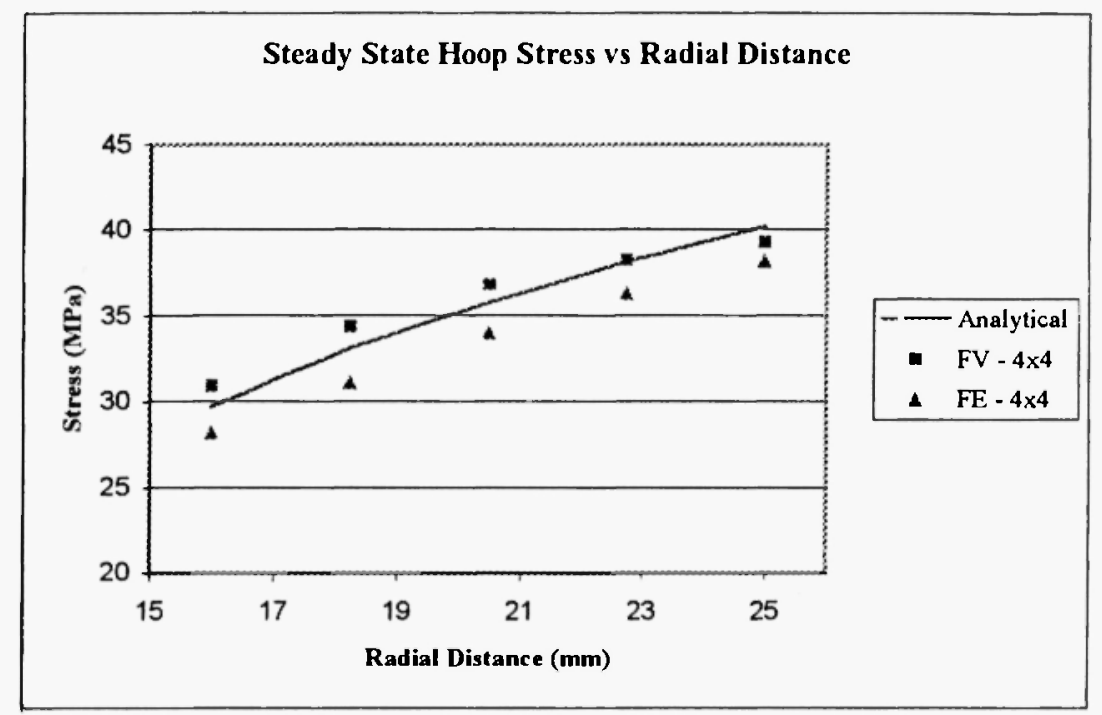

Figure 12 - Comparison of computed hoop stresses using FVM and FEM for uniform thick-walled tube.

\section{CONCLUSIONS}

A very accurate finite volume formulation and algorithm were described for elasticcreep analysis of axisymmetric components. Its verification and application to an internally corroded/eroded boiler tube were demonstrated. It was also shown that for the same level of mesh density, the present finite volume formulation was more accurate than a similarly formulated finite element method.

\section{REFERENCES}

Ivankovic, A and Williams J G, 1995, The Finite Volume Analysis of Linear Elastic Dynamic Fracture Problems, in Dynamic Fracture Mechanics, ed. Aliabadi, $\mathrm{M} \mathrm{H}$, Computational Mechanics Publications, Southampton, UK.

Onate, E, Cervera, M and Zienkiewicz, O C, 1994, A Finite Volume Format for Structural Mechanics applications, Int. J. Num. Meth In Eng., Vol 37, ppl81-201.

Owen D R J and Hinton E, 1980, Finite Elements in Plasticity: Theory and Practice, Pineridge Press Limited, UK

Zarrabi K, and Hosseini-Toudeshky H, 1995, Creep Life Assessment of Defect-free Components Under Uniform Load and Temperature. Int. J. Press. Vessels and Piping. Vol.62, No.2, ppl95-200.

Zarrabi K, Basu A and Rose 1, 1999, Application of the Finite Volume Method to Eroded/Corroded Boiler Tubes - Part I: Elastic Analysis, Proceedings of the $2^{\text {nd }}$ Australian Congress on Applied Mechanics, Canberra, Australia, 10-12 February 1999. 
